\title{
Uma Classe de Equações Diferenciais com Retardamento Aplicável ao Estudo de Redes Neurais
}

Selma Helena de Jesus

\author{
Orientação \\ Prof. Dr. Plácido Zoéga Táboas
}

Dissertação apresentada ao Instituto de Ciências Matemáticas de São Carlos USP, como parte dos requisitos para obtenção do título de Mestre em Matemática.

USP - São Carlos

agosto 1992 
Dedico a meu pai (in memorian),

à minha mãe,

ao Aldemir

e aos meus irmâos. 
Não poderá ser um verdadeiro matemático aquele que não for um pouco poeta.

Karl Theoder Wilhelm Weierstrass

... vida é fazer todo sonho brilhar ...

João Bosco 
Agradeço:

Ao Prof. Dr. Plácido Zoéga Táboas pela excelente orientação, paciência e amizade.

Aos professores do Instituto de Ciências Matemáticas de São Carlos e do Departamento de Matemática da Universidade Federal de São Carlos.

Aos técnicos do LDCC- ICMSC pela colaboração na edição deste trabalho.

À CNPq e CAPES, cujo auxílio através de bolsas, possibilitou a realização dos cursos de pós graduação.

Aos colegas da pós graduação, meus agradecimentos pela amizade e apoio nos momentos dificeis.

À minha familia pelo carinho e incentivo.

Enfim, a todos que direta ou indiretamente contribuíram para a realização deste trabalho. 
Abstract

We are concerned with the system

$$
\dot{u}_{i}(t)=-u_{i}(t)+\sum_{j=1}^{n} J_{i j} f\left(u_{j}(t-\tau)\right)
$$

$i=1,2, \ldots, n$, where $u_{i}$ is real, $\left(J_{i j}\right)$ is a $n \times n$ real matrix, $f(0)=0$ and the value $\beta=f^{\prime}(0)$ is the so called gain.

This is the C. M. Marcus and R. M. Westervelt mathematical model for an analog neural network with delay. We study periodic solutions of the system and bifurcation of such solutions when the delay $\tau$ plays the role of a parameter. 


\section{Resumo}

Estamos interessados no sistema

$$
\dot{u}_{i}(t)=-u_{i}(t)+\sum_{j=1}^{n} J_{i j} f\left(u_{j}(t-\tau)\right),
$$

$i=1,2, \ldots, n$, onde $u_{i}$ é real, $\left(J_{i j}\right)$ é uma $n \times n$ matriz real, $f(0)=0$ e o valor $\beta=f^{\prime}(0)$ é o assim chamado ganho.

Este é o modelo matemático de C. M. Marcus e R. M. Westervelt para uma rede neural analógica com retardamento. Estudamos soluçōes periódicas do sistema e bifurcação de tais soluções quando o retardamento $\tau$ desempenha o papel de um parâmetro. 


\section{Conteúdo}

Introdução $\quad$ ii

1 Preliminares 1

2 Um problema de bifurcação de soluções 6

2.1 Um modelo matemático para redes neurais com retardamento . . 6

2.2 Bifurcações em termos da equação linearizada $\ldots . . . . . . .77$

3 Região de estabilidade $\quad 15$

4 Um caso de matriz de conexão não simétrica 23

4.1 Um critério de estabilidade em termos do ganho . . . . . . . . . 23

4.2 Um critério de estabilidade em termos do retardamento . . . . . . 33

$\begin{array}{lr}\text { Bibliografia } & \mathbf{3 4}\end{array}$ 


\section{Introdução}

Além do interesse puramente matemático, dentro do qual melhor se enquadra este trabalho, as Equaçōes Diferenciais Funcionais com retardamento têm tido grande sucesso em modelos determinísticos. Uma grande incidência é observada nas áreas biológicas, em epidemiologia, dinâmica das populações, etc...

Conforme [3] e [6], citados em [7], redes neurais conectadas simetricamente, modeladas em tempo contínuo, não oscilam. Na linha de [7] estudaremos um modelo mais realistico levando em conta retardamentos no tempo, resultantes de capacitâncias e indutâncias parasíticas, usualmente negligenciadas.

Após algumas mudanças de variáveis e da escala do tempo, o modelo proposto por C. M. Marcus e R. M. Westervelt se traduz no sistema

$$
\dot{u}_{i}(t)=-u_{i}(t)+\sum_{j=1}^{n} J_{i j} f\left(u_{j}(t-\tau)\right),
$$

$i=1,2, \ldots, n$, onde $u_{i}$ é real, $\tau>0,\left(J_{i j}\right)$ é uma matriz real simétrica, $f(0)=0$ e $f^{\prime}(0)=\beta$. O parâmetro $\beta$ é chamado ganho. Nosso propósito é encontrar critérios de estabilidade para a solução trivial de (0.1).

No capítulo 1 colocamos fatos da teoria geral das equaçóes diferenciais funcionais com retardamento, contidos em [4], além de resultados da teoria de estabilidade no sentido Liapunov e de Análise Funcional que são usados no decorrer do trabalho.

Começamos realmente nosso estudo no capítulo 2. Nesse capítulo, observando que estudar o sistema (0.1) é equivalente a estudar a equação

$$
\dot{x}(t)=-x(t)+\lambda f(x(t-\tau)),
$$

onde $\lambda \in \mathbf{R}$ é autovalor da matriz $\left(J_{i j}\right)$, linearizamos a equação (0.2), mostrando a existência de bifurcações com o aparecimento de soluções periódicas não constantes, bem como pontos fixos não nulos, tomando $\lambda$ como parâmetro. A condição $\lambda \in \mathbf{R}$ é devido ao fato de $\left(J_{i j}\right)$ ser simétrica. 
No capítulo 3 encontramos uma região de estabilidade no $\lambda$ - plano complexo, para o parâmetro "ganho" fixado arbitrariamente, estudamos a variação dessa região quando o retardamento varia.

No capítulo 4 estudamos o modelo de C. M. Marcus e R. M. Westervelt de modo a incluir matrizes de conexão não simétricas. Mais precisamente esse estudo inclui matrizes de conexão cujas formas canônicas são diagonais de blocos do tipo

$$
\left[\begin{array}{cc}
0 & \lambda \\
-\lambda & 0
\end{array}\right] \text {. }
$$

Obtemos então critérios de estabilidade em termos do retardamento e do ganho.

Com isso concluimos que redes neurais modeladas em tempo contínuo com conexões simétricas não oscilam quando os neurônios têm resposta rápida, mas oscilam quando a defasagem entre o "input" e o "output" de cada neurônio se torna maior do que um certo valor crítico. 


\section{Capítulo 1}

\section{Preliminares}

Consideremos $\tau \geq 0$ um número real dado, o espaço $\mathbf{n}$-dimensional $\mathbf{R}^{\mathbf{n}}$ sobre os reais com a norma euclidiana $|\cdot|$ e $C\left([c, d], \mathbf{R}^{n}\right)$ o espaço de Banach das aplicaçóes contínuas do intervalo $[c, d]$ tomando valores em $\mathbf{R}^{n}$, munido da topologia da convergência uniforme.

Seja $[c, d]=[-\tau, 0]$ e $C=C\left([-\tau, 0], \mathbf{R}^{n}\right)$ onde a norma de um elemento $\phi \in C$ é dada por

$$
|\phi|=\sup _{-\tau \leq \theta \leq 0}|\phi(\theta)|
$$

Definição 1.1 Sejam $\sigma \in \mathbf{R}, A \geq 0$ e $x \in C\left([\sigma-\tau, \sigma+A], \mathbf{R}^{n}\right)$. Para cada $t \in[\sigma, \sigma+A]$, definimos a função $x_{t} \in C$ por

$$
x_{t}(\theta)=x(t+\theta)
$$

onde $-\tau \leq \theta \leq 0$.

Definição 1.2 Sejam $D$ um subconjunto de $\mathbf{R} \times C, F: D \rightarrow \mathbf{R}^{n}$ e denotemos com "." a derivada à direita com relação a $t$. A equação

$$
\dot{x}(t)=F\left(t, x_{t}\right)
$$

é dita uma equação diferencial funcional retardada sobre $D$ e será denotada por $\operatorname{EDFR}(\boldsymbol{F})$.

Definição 1.3 Se existe $\sigma \in \mathbf{R}$ e $A>0$ tal que $x \in C\left([\sigma-\tau, \sigma+A), \mathbf{R}^{n}\right)$, $\left(t, x_{t}\right) \in D$ e $x(t)$ satisfaz a equação (1.1) para $t \in[\sigma, \sigma+A)$, diz-se que $x$ é solução da equação (1.1). 
Definição 1.4 Dado $\sigma \in \mathbf{R}, \phi \in C$, dizemos que $x(\sigma, \phi)$ é solução da equação (1.1) com valor inicial $\phi$ em $\sigma$ se:

1. existe $A>0$ tal que $x(\sigma, \phi)$ é solução da equação (1.1) em $[\sigma-\tau, \sigma+A)$ e 2. $x_{\sigma}(\sigma, \phi)=\phi$.

Dizemos que a equação (1.1) é linear se $F(t, \phi)=L(t, \phi)+h(t)$, onde $L(t, \phi)$ é linear em $\phi$; linear homogênea se $h=0$ e linear não homogênea se $h \neq 0$. Dizemos que a equação (1.1) é autônoma se $F(t, \phi)=g(\phi)$, onde $g$ não depende de $t$.

A teoria das equaçóes diferenciais funcionais retardadas é inspirada na teoria das equaçôes diferenciais ordinárias, as quais são um tipo particular da equação (1.1), bastando tomar $\tau=0$.

Considerando problemas de valor inicial, ressaltemos que, como no caso das equações ordinárias, aqui também vale um teorema de existência e unicidade de soluções sob a hipótese de continuidade da $F$. Se $F$ for, além disso, localmente lipschitziana em $\phi$, temos unicidade de solução. Propriedades de extensão de soluçôes são válidas, com a ressalva de serem estabelecidos à direita.

Lema 1.1 Sejam $f: \mathbf{R} \rightarrow \mathbf{R}$ uma função contínua e $\lambda$ um parâmetro real. Para qualquer função inicial $\varphi \in C$, uma solução $x(0, \phi)$ da equação com retardamento $\dot{x}(t)=-x(t)+\lambda f(x(t-\tau))$, existe para todo $t \geq 0$.

Prova: O lema segue da representação implícita

$$
\left\{\begin{array}{l}
x(t)=\phi(0) e^{-t}+\int_{0}^{t} \lambda f(x(s-\tau)) e^{-(t-s)} d s, t \geq 0 \\
x_{0}=\phi
\end{array}\right.
$$

Seja $L$ uma função linear contínua de $C$ em $\mathbf{R}^{n}$, o que implica que existe uma matriz $n \times n, \eta(\theta),-\tau \leq \theta \leq 0$, cujos elementos são de variação limitada tal que

$$
L(\phi)=\int_{-\tau}^{0}[d \eta(\theta)] \phi(\theta), \phi \in C .
$$

A equação característica de $\dot{x}(t)=L\left(x_{t}\right)$ é dada por

$$
\operatorname{det} \Delta(s)=0, \Delta(s)=s I-\int_{-\tau}^{0} e^{s \theta} d \eta(\theta) .
$$


A equação característica para o caso particular de uma equação diferencial diferença linear homogênea com coeficientes constantes é obtida procurando soluções não triviais da forma $e^{s t} c$ onde $c$ é constante. Por exemplo, a equação escalar

$$
\dot{x}(t)=A x(t)+B x(t-\tau)
$$

tem uma solução não trivial da forma $e^{s t} c$ se, e somente se,

$$
h(s) \stackrel{\text { def }}{=} s-A-B e^{-s \tau}=0 .
$$

O livro de Bellman e Cooke [2] já se tornou uma referência clássica para o estudo de equações do tipo (1.2). Resultados significativos sobre o assunto também são devidos a Hayes [5]. As raizes $s$ de (1.2) são chamadas raizes características.

O estudo das possíveis mudanças na estrutura das órbitas de uma equação diferencial, dependendo de parâmetros variáveis, é chamado teoria da bifurcação. Para sabermos da existência de bifurcações, estudamos o sinal da parte real das raizes características.

Quando, com a variação do parâmetro, passamos a ter uma solução periódica não constante, temos uma bifurcação de Hopf, o que corresponde a um par de raizes caracteristicas complexas cruzar o eixo imaginário, de acordo com as hipóteses do Teorema 1.2.

Quando, com a variação do parâmetro, passamos a ter outras duas soluções constantes, além da solução trivial, temos uma bifurcação do tipo "pitchfork", o que corresponde a uma raiz característica $s$ real cruzar o eixo imaginário.

O teorema a seguir está contido em [2] e pode ser encontrado também no Apêndice de [4].

Teorema 1.1 Todas as raizes da equação $(z+\zeta) e^{z}+\gamma=0$, onde $\zeta$ e $\gamma$ são reais, têm parte real negativa se, e somente se,

$$
\begin{gathered}
\zeta>-1, \\
\zeta+\gamma>0, \\
\gamma<\xi \operatorname{sen} \xi-\zeta \cos \xi
\end{gathered}
$$

onde $\xi$ é a raiz da equą̧ão $\xi=-\zeta \tan \xi, 0<\xi<\pi$, se $\zeta \neq 0$ e $\xi=\pi / 2$ se $\zeta=0$. 
Consideremos uma família a um parâmetro de $\operatorname{EDFR}(F)$ da forma

$$
\dot{x}(t)=F\left(\alpha, x_{t}\right),
$$

onde $F(\alpha, \phi)$ tem primeira e segunda derivadas contínuas em $\alpha, \phi$, para $\alpha \in \mathbf{R}$, $\phi \in C$ e $F(\alpha, 0)=0$ para todo $\alpha$.

Seja $L: \mathbf{R} \times C \rightarrow \mathbf{R}^{n}$ dada por

$$
L(\alpha) \psi=F_{\phi}(\alpha, 0) \psi,
$$

onde $F_{\phi}(\alpha, 0)$ é a derivada de $F(\alpha, \phi)$ com respeito a $\phi$ em $\phi=0$.

Considere as seguintes hipóteses:

$\left(H_{1}\right)$ a $\operatorname{EDFR}(L(0))$ tem uma raiz caracteristica imaginária pura simples $s_{0}=i b_{0} \neq 0$ e toda raiz caracteristica $s_{j} \neq s_{0}, \bar{s}_{0}$, satisfaz $s_{j} \neq m s_{0}$ para todo inteiro $m$.

$\left(\mathbf{H}_{2}\right) \Re s^{\prime}(0) \neq 0$, onde a linha representa a derivada de $s$ em relação a $\alpha$.

As hipóteses $\left(\mathrm{H}_{1}\right)$ e $\left(\mathrm{H}_{2}\right)$ implicam que existem soluções periódicas não constantes da equação (1.3) para $\alpha$ pequeno, as quais têm período próximo à $2 \pi / b_{0}$.

É o teorema enunciado a seguir conhecido como Teorema da Bifurcação de Hopf, que fornece os detalhes dessa afirmação.

A formulação apresentada aqui é simplificada de modo a melhor atender os nossos propósitos. Está contida em [4, páginas 246-247].

Teorema 1.2 Suponhamos $F(\alpha, \phi)$ continuamente diferenciável em relação à $\alpha, \phi, F(\alpha, 0)=0$ para todo $\alpha, e$ as hipóteses $\left(H_{1}\right)$ e $\left(H_{2}\right)$ satisfeitas. Então existem constantes $a_{0}>0, \alpha_{0}>0, \delta_{0}>0$, funçöes continuamente diferenciáveis em $a, \alpha(a) \in \mathbf{R}, \omega(a) \in \mathbf{R}$ e $x^{*}(a) \omega(a)$-periódica, para $|a|<a_{0}$, de modo que $x^{*}(a)$ $\dot{e}$ uma solução da equação (1.3). Além disso, para $|\alpha|<\alpha_{0},\left|\omega-\left(2 \pi / b_{0}\right)\right|<\delta_{0}$, as soluções $x^{*}$ são as únicas $\omega$-periódicas da equação (1.3) com $\left|x_{t}\right|<\delta_{0}$, exceto por uma translação de fase.

Suponhamos que, sobre as hipóteses do Teorema $1.2, \alpha=0$ seja o primeiro valor do parâmetro $\alpha$ onde $\left(H_{1}\right),\left(H_{2}\right)$ valem, isto é, $\alpha<0$ implica $\Re s<0$ para toda raiz característica $s$. Nesse caso, quando $\alpha<0$, a origem é uma solução assintoticamente estável de (1.3) e zero se caracteriza como um valor que, ao ser atingido por $\alpha$, provoca uma perda de estabilidade. 
Em relação à equação (1.1), consideremos as seguintes hipóteses:

1. o segundo membro da equação é uma função completamente contínua e

2. vale alguma condiçâo de unicidade relativamente ao problema de função inicial.

As seguintes definições de estabilidade são dadas em relação ao ponto de equilibrio $x=0$, mas podem ser facilmente estendidas a uma solução qualquer $x(t), t \geq 0$.

Definição 1.5 Dizemos que $x=0$ é estável se, dados $\epsilon>0$ e $\sigma \geq 0$, existe $\delta=\delta(\epsilon, \sigma)$ tal que $\|\varphi\|<\delta$ e $t \geq \sigma$ implicam $\left\|x_{t}(\sigma, \varphi)\right\|<\epsilon$.

Definição 1.6 Dados $\epsilon>0$ e $\sigma \geq 0$, se existe $\delta=\delta(\epsilon)>0$ tal que, $\|\varphi\|<\delta e$ $t \geq \sigma$ implicam $\left\|x_{t}(\sigma, \varphi)\right\|<\epsilon$, dizemos que $x=0$ é uniformemente estável.

Definição 1.7 Se a condição de estabilidade está satisfeita e a todo $\sigma \geq 0$ corresponde $\rho=\rho(\sigma)>0$ tal que $\|\varphi\|<\rho$ implica $x(t, \sigma, \varphi) \rightarrow 0$ quando $t \rightarrow \infty$, dizemos que $x=0$ é assintoticamente estável.

O seguinte Lema sobre estabilidade está contido em resultados mais gerais, veja [4, Seç̧ão 10.1].

Lema 1.2 Se nenhuma raiz característica $s$ de (1.2) tiver $\Re s=0$, então uma condição necessária e suficiente para que zero seja assintoticamente estável, é que não exista raiz característica $s$ com $\Re(s)>0$. 


\section{Capítulo 2}

\section{Um problema de bifurcação de soluções}

\subsection{Um modelo matemático para redes neurais com retardamento}

O modelo de C.M. Marcus e R.M. Westervelt para redes neurais, levando em conta defasagem entre o "input" e o "output" em cada neurônio, traduz-se matematicamente no sistema de equações diferenciais com retardamento

$$
C_{i} \dot{v}_{i}\left(t^{\prime}\right)=-\frac{1}{R_{i}} v_{i}\left(t^{\prime}\right)+\sum_{j=1}^{n} T_{i j} f_{j}\left(v_{j}\left(t^{\prime}-\tau_{j}^{\prime}\right)\right) .
$$

A variável $v_{i}$, representa a voltagem do "input" no i-ésimo neurônio, $i=1,2, \ldots, n$.

Os retardamentos $\tau_{j}^{\prime}, j=1, \ldots, n$, representam o tempo de resposta de cada neurônio. As funções $f_{j}$ são chamadas funções transferência e, assim como C.M. Marcus e R.M. Westervelt, faremos a simplificação $\tau_{j}=\tau, f_{j}=f, j=1, \ldots, n$. A função transferência $f$ é suposta "sigmoidal", saturando em \pm 1 com inclinação máxima em $v=0$, ou seja, $f$ é pelo menos $C^{1}$, ímpar, $f^{\prime}$ é estritamente decrescente em $\mathbf{R}_{+}$com $\lim _{v \rightarrow \infty} f(v)=1$, e $f^{\prime}(0)=\max _{v \in \mathbf{R}} f^{\prime}(v)$. Por simplicidade, vamos supor em todo este trabalho que $f$ é suficientemente regular, de modo que existam todas as suas derivadas que necessitarmos.

A matriz $\left(T_{i j}\right)$ é chamada matriz de conexão e suas entradas $T_{i j}$ têm magnitude inversamente proporcional a certas resistências $R_{i j}$ inerentes à construção da rede. 
Após algumas simplificações que envolvem a consideração de neurônios idênticos, o sistema se reduz a

$$
R C \dot{v}_{i}\left(t^{\prime}\right)=-v_{i}\left(t^{\prime}\right)+R \sum_{j=1}^{n} T_{i j} f\left(v_{j}\left(t^{\prime}-\tau^{\prime}\right)\right) .
$$

Façamos as seguintes mudanças de variáveis:

$$
t=\frac{t^{\prime}}{R C}, \tau=\frac{\tau^{\prime}}{R C} \text { e } J_{i j}=R T_{i j}
$$

Em termos das novas variáveis, temos

$$
\dot{u}_{i}(t)=-u_{i}(t)+\sum_{j=1}^{n} J_{i j} f\left(u_{j}(t-\tau)\right) .
$$

\subsection{Bifurcações em termos da equação lineari- zada}

Nessa secção introduzimos uma análise de estabilidade linear na origem, isto é, onde todos os neurônios têm voltagem zero. Nosso objetivo é mostrar o aparecimento de soluções periódicas não constantes, bem como pontos fixos não nulos do sistema

$$
\dot{u}_{i}(t)=-u_{i}(t)+\sum_{j=1}^{n} J_{i j} f\left(u_{j}(t-\tau)\right),
$$

onde $i=1, \ldots, n, u_{i}$ é real, $\tau>0,\left(J_{i j}\right)$ é uma matriz real simétrica, $f \dot{e}$ "sigmoidal $^{\prime \prime}, f^{\prime}(0)=\beta>0, f(0)=0$.

Sendo a matriz $\left(J_{i j}\right)$ simétrica, é diagonalizável, portanto, podemos olhar o sistema em $n$ subespaços de dimensão 1 . Ou seja, o problema é equivalente ao da equação escalar

$$
\dot{x}(t)=-x(t)+\lambda f(x(t-\tau))
$$

onde $\lambda$ é autovalor da matriz $\left(J_{i j}\right)$ chamada matriz de conexão. Chamaremos $\beta$ de ganho e os autovalores da matriz $\left(J_{i j}\right)$ de autovalores de conexão para evitar confusão com as raízes características da equação linearizada.

Vamos obter a equação característica da parte linear da equação (2.5). Por Taylor, em uma vizinhança da origem temos,

$$
f(x)=0+\beta x+o(x) \text {. }
$$


Substituindo na equaçắo (2.5) temos,

$$
\dot{x}(t)=-x(t)+\lambda \beta x(t-\tau)+o(x) .
$$

Logo a parte linear é

$$
\dot{x}(t)=-x(t)+\beta \lambda x(t-\tau) .
$$

Assim uma condição necessária e suficiente para a existência de uma solução não trivial da equação (2.6) da forma $x(t)=e^{e t} c$, onde $c \in \mathbf{R}$ é que $c$ seja uma solução não nula de

$$
s e^{s t} c=-e^{s t} c+\beta \lambda e^{o(t-\tau)} c,
$$

ou seja,

$$
\left[(s+1) e^{s \tau}-\beta \lambda\right] c=0,
$$

a qual nos leva à equação característica da equação (2.6),

$$
(s+1) e^{s \tau}=\beta \lambda,
$$

e essa, após uma mudança do parâmetro $\beta, \alpha=\beta \lambda e^{\tau}$ e uma translação de $s$, $\mu=s+1$, torna-se

$$
\mu e^{\mu \tau}=\alpha .
$$

Vamos mostrar que existe uma bifurcação de Hopf e uma bifurcação do tipo "pitchfork". Para fazer isso, precisaremos de informaçōes sobre o comportamento dos zeros da equação (2.8).

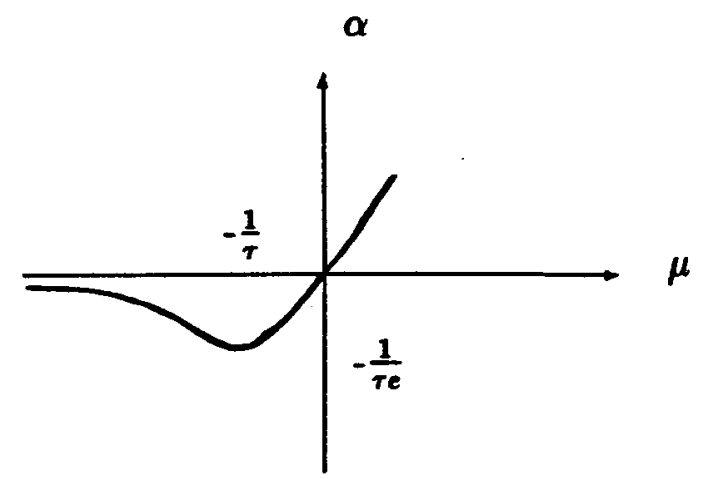

Figura 2.1: $\alpha=\mu e^{\mu \tau}$ 
Vamos agora determinar condições sob as quais a equação (2.8) tenha raízes reais. Seja $\alpha=\rho(\mu)=\mu e^{\mu \tau}$. Então $\rho^{\prime}(\mu)=(1+\mu \tau) e^{\mu \tau}$ e, portanto, $\rho^{\prime}(\mu)<0$, quando $-\infty<\mu<-1 / \tau, \rho^{\prime}(-1 / \tau)=0$ e $\rho^{\prime}(\mu)>0$, quando $-1 / \tau<\mu<\infty$. 0 gráfico de $\rho(\mu)=\mu e^{\mu \tau}$ está esboçado na figura (2.1).

Consequentemente, $\rho(\mu)$ é mínimo em $\mu=-1 / \tau, \rho(-1 / \tau)=-1 / \tau e$. Portanto, a equação (2.8) tem raizes reais para $\alpha>-1 / \tau e$.

Observação $2.1 \mu$ é raiz de (2.8) se, e somente se, sua complexa conjugada $\bar{\mu}$ também o é. Portanto podemos restringir nosso estudo ao semiplano superior $\Im(\mu)>0$.

Lema 2.1 Se ã, $0<\tilde{a}<1$, é uma raiz real de $\mu e^{\mu \tau}=\alpha, 0<\alpha<e^{\tau}$, então toda raiz $\mu=a+b i, b \neq 0$, satisfaz $\Re \mu \leq \tilde{a}$.

Prova: Se $\mu=a+b i, a, b \in \mathbf{R}$ é uma rajz de (2.8), então $\mu$ deve satisfazer a condição $\sqrt{a^{2}+b^{2}} e^{a \tau}=|\alpha|$, portanto $\left(a^{2}+b^{2}\right) e^{2 a r}=\alpha^{2}$. Assim,

$$
b=\left[\left(\alpha e^{-a \tau}-a\right)\left(\alpha e^{-a \tau}+a\right)\right]^{1 / 2} .
$$

Para que $b$ esteja bem definido é necessário que $\alpha e^{-a \tau}-a \geq 0$. Então, definamos $\rho(a)=\alpha e^{-a \tau}-a$. Logo, $\rho(0)=\alpha, \rho^{\prime}(a)=-\left(\tau \alpha e^{-a \tau}+1\right)<0$.

Temos que $\rho(a) \geq 0$ para $0<a<\tilde{a}$, onde ã é tal que $\rho(\tilde{a})=0$, ou seja, $\tilde{a} e^{\tilde{a} \tau}=\alpha$. Logo, $\Re \mu \leq \tilde{a}$. Ainda mais, $d b / d a=-(1 / b)\left(\alpha^{2} \tau e^{-2 a \tau}+a\right)<0$.

Lema 2.2 Seja $\alpha_{0}$ dado por $\alpha_{0}=-b_{0} e^{\tau} / \operatorname{sen}\left(b_{0} \tau\right)<0$, onde $b_{0}$ é a única solução de $b \cot (b \tau)=-1$ em $(0, \pi / \tau)$, portanto $\alpha_{0}<-\pi / 2 \tau$. Então valem as seguintes afirmaçōes:

1. Se $\alpha>e^{\tau}$, existe uma raiz real de (2.8) maior que 1 .

2. Se $0<\alpha<e^{\tau}$, existe uma raiz real ã de (2.8) menor que 1 e toda raiz $\mu$ satisfaz $\Re \mu \leq \tilde{a}$.

3. Se $\alpha_{0}<\alpha<0$, toda solução da equação (2.8) tem parte real menor que 1 e se $\alpha<\alpha_{0}$, existe uma raiz de (2.8) da forma $\mu(\alpha)=a(\alpha)+i b(\alpha)$ a qual é continuamente diferenciável em $\alpha$ e satisfaz $0<b(\alpha)<\pi / \tau, b\left(\alpha_{0}\right)>0$, $a\left(\alpha_{0}\right)=1$ e $a(\alpha)>1$ para $\alpha<\alpha_{0}$. 
Prova: A importância de se comparar o valor de $\Re \mu$ com 1 é que, com a mudança de variável $s+1=\mu$ dada acima, a condição de estabilidade dada por $\Re s<0$ torna-se $\Re \mu<1$.

1. De acordo com a discussão da equação (2.8) anterior ao lema 2.1 , temos que $\rho(1)=e^{\tau}$ e que, para $\alpha>e^{\tau}$, existe raiz real $\mu>1$ de (2.8).

2. Segue do lema 2.1 e das considerações anteriores a ele.

3. Multiplicando a equação (2.8) por $\tau$ obtemos

$$
\tau \mu e^{\mu \tau}=\tau \alpha .
$$

Pelo teorema 1.1 do capítulo 1 temos $\zeta=0>-1, \gamma=-\alpha \tau>0$, pois $\alpha<0, \zeta+\gamma=-\alpha \tau$.

Como $\zeta=0$ temos que $\xi=\pi / 2$, o que implica que $\gamma<\pi / 2$. Como $\gamma=-\alpha \tau$, concluímos que $\alpha>-\pi / 2 \tau$. Então para $-\pi / 2 \tau<\alpha<0$, temos que as raizes de (2.8) têm parte real negativa.

Se $\alpha<-1 / \tau e$, as raízes da equação (2.8) são da forma $\mu=a+b i, b \neq 0$, e portanto satisfazem

$$
(a+b i) e^{\tau(a+b i)}=\alpha,
$$

ou seja,

$$
\left\{\begin{array}{l}
e^{a \tau}[a \cos (b \tau)-b \operatorname{sen}(b \tau)]=\alpha \\
e^{a \tau}[a \operatorname{sen}(b \tau)+b \cos (b \tau)]=0 .
\end{array}\right.
$$

Da segunda equação obtemos $a=-b \cot (b \tau)$. Substituindo $a$ na primeira equação do sistema (2.11) obtemos

$$
\alpha=\frac{-b e^{-b \tau \cot (b \tau)}}{\operatorname{sen}(b \tau)} \stackrel{\text { def }}{=} \Gamma(b) .
$$

Vamos considerar $\Gamma(b)$ para $0<b<\pi / \tau$. É claro que $\Gamma(b)$ é contínua e $\Gamma(b)<0$.

Consideremos as faixas

$$
T_{2 k}=\left\{\mu=a+b i: \frac{2 k \pi}{\tau}<b<\frac{(2 k+1) \pi}{\tau}\right\}, k=0,1, \ldots
$$

e

$$
T_{2 k-1}=\left\{\mu=a+b i: \frac{(2 k-1) \pi}{\tau}<b<\frac{2 k \pi}{\tau}\right\}, k=1,2, \ldots
$$


Sendo $\alpha<0$, não é difícil ver que a equação (2.12) é incompatível com a condição

$$
\frac{(2 k-1) \pi}{\tau}<b<\frac{2 k \pi}{\tau}, k=1,2, \ldots
$$

Assim, não existem raízes de (2.8) nas faixas $T_{2 k-1}$.

Com algum trabalho de rotina pode-se preencher os detalhes dos seguintes cálculos.

$$
\begin{aligned}
\frac{d \Gamma}{d b}= & \frac{\left\{-b e^{-b \tau \cot (b \tau)}\left[-\tau \cot (b \tau)+b \tau^{2} \operatorname{cossec}^{2}(b \tau)\right]-e^{-b \tau \cot (b \tau)}\right\} \operatorname{sen}(b \tau)}{\operatorname{sen}^{2}(b \tau)}+ \\
& +\frac{\tau b e^{-b \tau \cot (b \tau)} \cos (b \tau)}{\operatorname{sen}^{2}(b \tau)}= \\
=-e^{-b \tau \cot (b \tau)}\left\{[\operatorname{sen}(b \tau)-b \tau \cos (b \tau)]^{2}-b^{2} \tau^{2} \cos ^{2}(b \tau)+b^{2} \tau^{2}\right\} & \frac{\operatorname{cossec}(b \tau)}{\operatorname{sen}^{2}(b \tau)}
\end{aligned}
$$

Como $0<b \tau<\pi$ e $0<\cos ^{2} b \tau<1$, temos que $\operatorname{cossec}(b \tau)>0$ e $b^{2} \tau^{2} \cos ^{2} b \tau<b^{2} \tau^{2}$. Portanto $d \Gamma / d b<0$.

Ainda mais, $\Gamma(b) \rightarrow-\infty$ quando $b \rightarrow \frac{\pi}{\tau}-$ e $\Gamma(b) \rightarrow-\frac{1}{\tau e}$ quando $b \rightarrow 0+$.

Portanto existe exatamente um valor de $b$, vamos dizer $b=b(\alpha)$, $0<b(\alpha)<\pi / \tau$, para o qual $\Gamma(b(\alpha))=\alpha$, se $\alpha<-\frac{1}{\tau e}$.

Assim, $a(\alpha)=-b(\alpha) \cot [b(\alpha) \tau]$. As funçóes $b(\alpha)$ e $a(\alpha)$ são claramente diferenciáveis em $\alpha, 0<b<\pi / \tau$.

Afirmamos que as raizes imaginárias puras de (2.8) para $0<b<\pi / \tau$ são $\mu=\frac{\pi}{2 \tau} i$ e ocorrem para $\alpha=-\frac{\pi}{2 \tau}$.

De fato, fazendo $\mu=b i$ no sistema (2.11) obtemos

$$
\left\{\begin{array}{l}
\operatorname{sen}(b \tau)=-\alpha / b \\
\cos (b \tau)=0 .
\end{array}\right.
$$

Como temos $0<b \tau<\pi \mathrm{e}-\alpha / b>0$, pois $\alpha<0$, concluímos que $b=\pi / 2 \tau$, $\Gamma(\pi / 2 \tau)=-\pi / 2 \tau, b(-\pi / 2 \tau)=\pi / 2 \tau$ e $a(-\pi / 2 \tau)=0$.

Como $d \Gamma / d b<0$, pelo teorema da função inversa temos que $d b / d \alpha<0$ e portanto $b(\alpha)$ é decrescente.

Assim $\alpha<-\pi / 2 \tau$ se, e somente se, $b(\alpha)>\pi / 2 \tau$ que por sua vez, implica $b(\alpha) \tau>\pi / 2$. Logo $\cot [b(\alpha) \tau]<0$. Pela segunda equação do sistema $(2.11)$ concluimos, para $\alpha<-\pi / 2 \tau$, que

$$
\frac{d a}{d \alpha}=\left\{-\cot [b(\alpha) \tau]+b(\alpha) \tau \operatorname{cossec}^{2}[b(\alpha) \tau]\right\} \frac{d b}{d \alpha}<0 .
$$


Então, para $\alpha_{0}<\alpha<-\pi / 2 \tau$, temos que toda solução da equação (2.8) tem parte real entre $0 \mathrm{e} 1$. Como $a(-\pi / 2 \tau)=0, a\left(\alpha_{0}\right)=1$ e $a(\alpha)$ é função decrescente, temos que $\alpha_{0}<-\pi / 2 \tau$. Também $b\left(\alpha_{0}\right)>0$ e $a(\alpha)>1$ para $\alpha<\alpha_{0}$.

Lema 2.3 $S e \mu_{2 k}=a_{2 k}+b_{2 k} i \in T_{2 k}, k=0,1, \ldots$ são raizes da equação (2.8), então suas partes reais satisfazem $a_{0}>a_{2}>\ldots \rightarrow-\infty$.

Prova: Se $\mu_{2 k}=a+b i$, a equação (2.8) implica que as raízes $\mu_{2 k}, k=0,1, \ldots$ devem satisfazer a condição

$$
\left(a^{2}+b^{2}\right) e^{2 a \tau}=\alpha^{2}
$$

e essa, para $\alpha=-\pi / 2 \pi$, define $b>0$ como função de $a$ no intervalo $-\infty<a<0$. De fato,

$$
a^{2}+b^{2}=\frac{\pi^{2}}{4 \tau^{2}} e^{-2 a \tau},
$$

o que implica

$$
b=\left[\left(\frac{\pi}{2 \tau} e^{-a \tau}+a\right)\left(\frac{\pi}{2 \tau} e^{-a \tau}-a\right)\right]^{1 / 2} .
$$

Temos que verificar que

$$
G(a) \stackrel{\text { def }}{=} \frac{\pi}{2 \tau} e^{-a r}+a
$$

é positiva para $a \leq 0$. Mas $G(0)=\pi / 2 \tau, G^{\prime}(a)=-\frac{\pi}{2} e^{-a \tau}+1<0$ e, portanto $G(a)>0$ para $a \leq 0$. Como

$$
\frac{d b}{d a}=-\frac{1}{b}\left(\frac{\pi^{2}}{4 \tau} e^{-2 a \tau}+a\right)<0
$$

para $a \leq 0$, segue que os $a_{2 k}^{\prime} s$ são estritamente decrescentes em relação aos $b_{2 k}^{\prime} s$. Assim, a ordem especificada das partes reais das raízes ocorre pelo menos no caso em que $\alpha=-\pi / 2 \tau$.

Suponhamos que o lema seja falso para algum valor do parâmetro $\alpha$. Então recordando que cada $a_{2 k}$ é uma função contínua de $\alpha$, existe algum $\alpha=\hat{\alpha}$, para o qual a equação (2.8) tem raízes $\mu_{2 k}$ e $\mu_{2 l}$ tais que $a_{2 k}=a_{2 l}=a, k>l$. Mas, como $b_{2 k}>b_{2 l}$, a equação (2.13) leva-nos à contradição

$$
\left(a^{2}+b_{2 k}^{2}\right) e^{2 a \tau}=\left(a^{2}+b_{2 l}^{2}\right) e^{2 a \tau} .
$$

Assim, $a_{0}>a_{2}>\ldots$

Fazendo $a=a_{2 k}, b=b_{2 k}$ em (2.13), vemos que $a_{2 k} \rightarrow-\infty$ quando $k \rightarrow \infty$. 
A figura 2.2 mostra a localização das raizes características nas faixas $T_{2 k}, k=$ $0,1, \ldots$, de acordo com as provas dos lemas 2.2 e 2.3. Quando $\alpha$ varia de $-1 / \tau e$ até $-\infty$, as raízes percorrem as curvas indicadas, no sentido da parte real crescente.

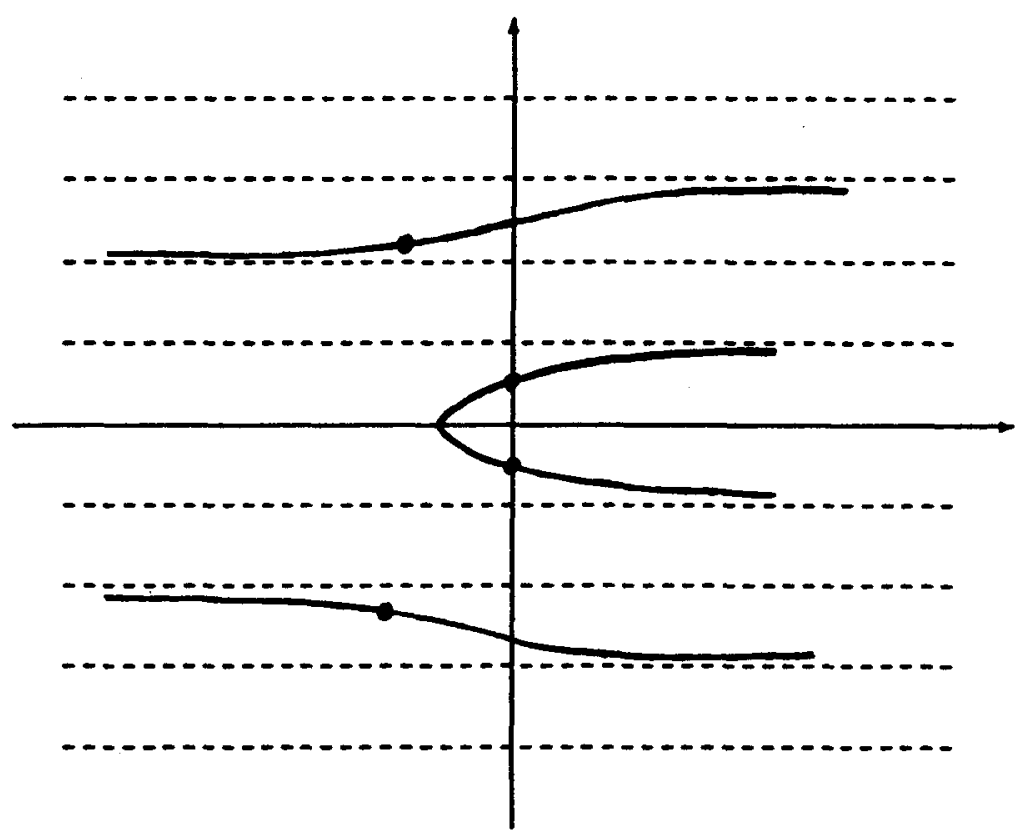

Figura 2.2: Lugar geométrico das raizes de (2.8)

Teorema 2.1 A equação (2.5) tem uma bifurcação do tipo "pitchfork" em $\alpha=e^{\tau}$ $e$ uma bifurcação de Hopf em $\alpha=-b_{0} e^{\tau} / \operatorname{sen}\left(b_{0} \tau\right)$, onde $b_{0}$ é a única raiz da equação $b \cot (b \tau)=1,0<b<\pi / \tau$.

Prova: Como é usual em resultados deste tipo, a prova se baseia na localização das raízes características da equação (2.8). A parte relativa à bifurcação de Hopf segue dos lemas 2.1, 2.2 e do teorema da bifurcação de Hopf 1.2.

Provemos a afirmação relativa à bifurcação do tipo "pitchfork". Se $\alpha>e^{\tau}$, então $\lambda>1 / \beta$, o que implica que $(\lambda f)^{\prime}(0)>1$. Assim, existem dois outros pontos fixos da função $\lambda f$, um em cada lado da origem (lembrando que $f$ é "sigmoidal"). Seja $\hat{x}$ um dos pontos fixos de $f$ e mostremos que $\hat{x}$ é solução estável da equação (2.5).

Seja $\epsilon>0$ dado e mostremos que existe $\delta>0$ tal que, se $\|\varphi-\hat{x}\|<\delta$ então $\|x(t, \varphi)-\hat{x}\|<\epsilon, t \geq 0$. 
Suponhamos temporariamente que exista $\epsilon>0$ de modo que, para todo $\delta, 0<\delta<\epsilon$, e todo $\varphi$ com $\|\varphi-\hat{x}\|<\delta$, tenhamos $x(t, \varphi) \notin(\hat{x}-\epsilon, \hat{x}+\epsilon)$. Então existe um primeiro $\tilde{t}$, tal que $x(\tilde{t})=\hat{x}+\epsilon$ ou $x(\tilde{t})=\hat{x}-\epsilon$. Vamos nos fixar no caso $x(\tilde{t})=\hat{x}+\epsilon$, uma vez que a outra alternativa pode ser tratada de modo completamente análogo. Assim,

$$
\dot{x}(\tilde{t})=-\hat{x}-\epsilon+\lambda f(x(\tilde{t}-\tau)) .
$$

Mas usando as condições de $f$ ser sigmoidal, podemos escrever

$$
\lambda f(x(\tilde{t}-\tau))<\lambda f(\hat{x}+\epsilon)<\hat{x}+\epsilon
$$

e, portanto $\dot{x}(\tilde{t})<0$, o que contradiz a escolha de $\tilde{t}$.

Logo $\hat{x}$ é estável.

Analogamente $-\hat{x}$ também é estável. 


\section{Capítulo 3}

\section{Região de estabilidade}

Quando a matriz de conexão $\left(J_{i j}\right)$ nã̀o é simétrica, os autovalores de conexão $\lambda$ podem ser complexos. Assim, nesse capítulo mostraremos a região de estabilidade no $\lambda$-plano complexo definida pela condição $\Re s<0$.

Quando $\tau=0$ a equação característica

$$
(s+1) e^{s \tau}=\beta \lambda
$$

torna-se polinomial

$$
s+1=\beta \lambda .
$$

Neste caso, o problema é consideravelmente mais simples e exaustivamente estudado na literatura. É sabido que a origem será assintoticamente estável se,

$$
\Re s=\beta \Re \lambda-1<0,
$$

ou seja,

$$
\Re \lambda<1 / \beta .
$$

Vamos nos concentrar no caso $\tau>0$.

Fazendo a seguinte mudança de variável $s+1 \stackrel{\text { def }}{=} \mu$ na equação (3.1) obtemos

$$
\mu e^{(\mu-1) \tau}=\beta \lambda .
$$

Com essa mudança de variável, a região de estabilidade dada pela condição $\Re s<0$ torna-se $\Re \mu<1$. Seja $\mu=a+b i$. Então a equação (3.2) se escreve 


$$
(a+b i) e^{(a-1+b i) \tau}=\beta \lambda
$$

ou seja,

$$
(a+b i) e^{a \tau} e^{-\tau}[\cos (b \tau)+i \operatorname{sen}(b \tau)]=\beta \lambda .
$$

Determinemos o contorno da região de estabilidade no plano $\lambda=A+B i$, fixando $a=\Re \mu=1$. Assim, esta equação fica

$$
(1+b i)[\cos (b \tau)+i \operatorname{sen}(b \tau)]=\beta A+i \beta B,
$$

ou seja,

$$
\left\{\begin{array}{l}
\beta A=\cos (b \tau)-b \operatorname{sen}(b \tau) \\
\beta B=b \cos (b \tau)+\operatorname{sen}(b \tau) .
\end{array}\right.
$$

Indicando com "." o produto interno usual, essas equações podem ser escritas equivalentemente na forma:

$$
\left\{\begin{array}{l}
\beta A=(1, b) \cdot[\cos (b \tau),-\operatorname{sen}(b \tau)] \stackrel{\text { def }}{=} \phi(b) \\
\beta B=(1, b) \cdot[\operatorname{sen}(b \tau), \cos (b \tau)] \stackrel{\text { def }}{=} \psi(b) .
\end{array}\right.
$$

Enquanto o vetor $(1, b), b \in \mathbf{R}$, percorre a reta $x=1$ no sentido crescente, $o$ vetor $[\cos (b \tau),-\operatorname{sen}(b \tau)]$ percorre sucessivamente a circunferência de centro $0 \mathrm{e}$ raio 1 no sentido horário. Nosso objetivo presente é descrever o lugar geométrico dos valores $\lambda=A+B i$ de modo que o sistema (3.4) esteja satisfeito para algum valor de $b$. Observemos inicialmente que $\phi(b)$ e $\psi(b)$ são, respectivamente, as coordenadas do vetor $(1, b)$ na direção dos vetores unitários $[\cos (b \tau),-\operatorname{sen}(b \tau)]$ e $[\operatorname{sen}(b \tau), \cos (b \tau)]$.

Desse modo, para $b=0$ temos $\phi(0)=1, \psi(0)=0$ e portanto, de acordo com (3.4), $A=1 / \beta$ e $B=0$.

Afirmação 3.1 Existe $b_{1}$ tal que $\phi\left(b_{1}\right)=0$.

De fato, para $b=0$ temos $\phi(0)=1>0$ e para $b=\pi / 2 \tau$ temos que $\phi(\pi / 2 \tau)=$ $-\pi / 2 \tau<0$. Pela continuidade da $\phi$, segue que existe $b_{1}$ tal que:

$$
0<b_{1}<\frac{\pi}{2 \tau} \text { e } \phi\left(b_{1}\right)=0 .
$$

O valor $b_{1}$ torna $\left(1, b_{1}\right)$ ortogonal à $\left[\cos \left(b_{1} \tau\right),-\operatorname{sen}\left(b_{1} \tau\right)\right]$. Logo, $\left(1, b_{1}\right)$ tem a direção do vetor $\left[\operatorname{sen}\left(b_{1} \tau\right), \cos \left(b_{1} \tau\right)\right]$. 
Assim, de acordo com a figura 3.1 , o valor $b_{1}$ é definido como a única solução entre 0 e $\pi / 2 \tau$ da equação

$$
b=\cot (b \tau) \text {. }
$$

Neste caso, novamente a figura 3.1 e a segunda equação de (3.4) fornecem

$$
\beta B=\sqrt{1+b_{1}^{2}},
$$

ou seja,

$$
B=(1 / \beta) \sqrt{1+b_{1}^{2}} .
$$

Desse modo, para $b=b_{1}$ temos $A=0$ e $B=(1 / \beta) \sqrt{1+b_{1}^{2}}$.

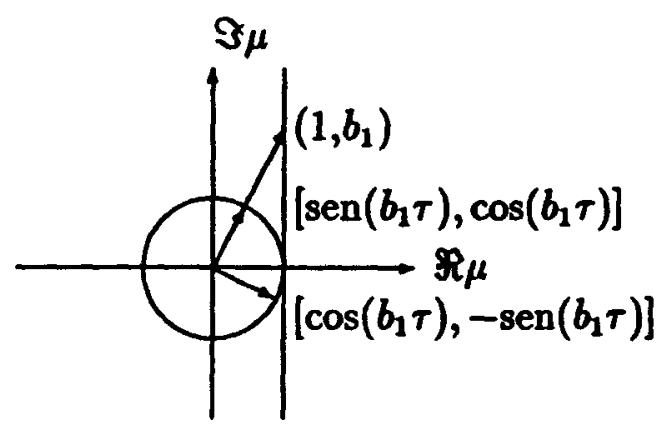

Figura 3.1: Representação de $b_{1}$

Afirmação 3.2 Existe $b_{2}>0$ tal que $\psi\left(b_{2}\right)=0$.

De fato, para $b=\pi / 2 \tau$ temos $\psi(\pi / 2 \tau)=1>0$ e para $b=\pi / \tau$ temos que $\psi(\pi / \tau)=-\pi / \tau<0$. Pela continuidade de $\psi$, segue que existe $b_{2}$ tal que:

$$
\frac{\pi}{2 \tau}<b_{2}<\frac{\pi}{\tau} \text { e } \psi\left(b_{2}\right)=0 .
$$

$O$ valor $b_{2}$ torna $\left(1, b_{2}\right)$ ortogonal à $\left[\operatorname{sen}\left(b_{2} \tau\right), \cos \left(b_{2} \tau\right)\right]$. Logo $\left(1, b_{2}\right)$ tem a direção do vetor $\left[\cos \left(b_{2} \tau\right),-\operatorname{sen}\left(b_{2} \tau\right)\right]$.

Assim, de acordo com a figura 3.2, o valor $b_{2}$ é definido como a única solução entre $\pi / 2 \tau$ e $\pi / \tau$ da equação

$$
b=-\tan (b \tau)
$$


Neste caso, observando que o segundo membro da primeira equação de (3.4) é negativo, novamente a figura $3.2 \mathrm{e}$ a primeira equação de (3.4) fornecem

$$
\beta A=-\sqrt{1+b_{2}^{2}}
$$

ou seja,

$$
A=-(1 / \beta) \sqrt{1+b_{2}^{2}} .
$$

Desse modo, para $b=b_{2}$ temos $A=-(1 / \beta) \sqrt{1+b_{2}^{2}}$ e $B=0$.

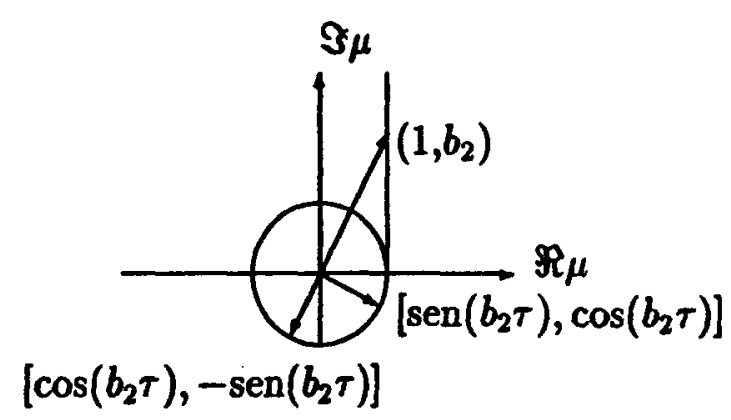

Figura 3.2: Representação de $b_{2}$

Afirmação 3.3 A curva definida no $\lambda$-plano complexo, $\lambda=A+B i$, pelas equações (3.4) é simétrica com relação ao eixo $A$.

De fato, se $\left(A_{0}, B_{0}\right)$ é o ponto dado em (3.4) correspondente a $b=b_{0}$, segue-se por verificação direta que $\left(A_{0},-B_{0}\right)$ é o ponto correspondente a $-b_{0}$.

Fixados $\beta>0$ e $\tau>0$, a curva $L(b)=(\phi(b), \psi(b))$ dada pelo sistema (3.4) para $b>0$, é o lugar geométrico dos autovalores de conexão $\lambda=A+B i$, tal que existe raiz característica $\mu$ da forma $\mu=1+b i$.

A curva é a espiral esboçada na figura 3.3, pois $|\lambda|$ é uma função crescente de b. A curva pontilhada corresponde à curva $L$ para $b<0$. 


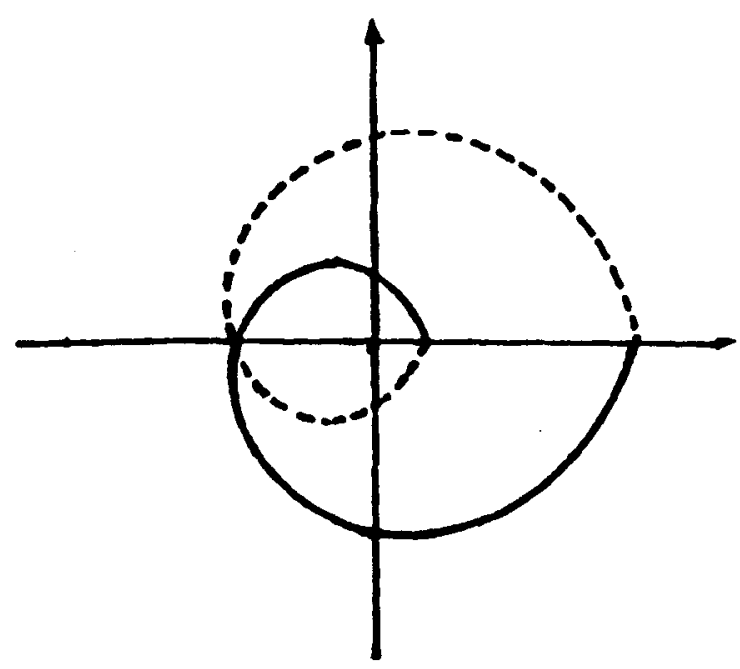

Figura 3.3: Lugar geométrico de $\lambda$ para $\Re \mu=1$

Lema 3.1 A região de estabilidade no $\lambda$-plano complexo é:

(i) para $\tau=0$, o semiplano à esquerda da reta $\Re \lambda=1 / \beta$;

(ii) para $\tau>0$, uma região limitada da forma de uma "gota", tal que:

(a) decresce e tende a um círculo, quando $\tau \rightarrow \infty$,

(b) cresce e tende ao semiplano $\Re \lambda<1 / \beta$, quando $\tau \rightarrow 0$.

Prova:

(i) Segue das consideraçôes feitas no início deste capítulo.

(ii) (a) Fazendo $\tau \rightarrow \infty$ em (3.5) e (3.8), temos que $b_{1} \rightarrow 0$ e $b_{2} \rightarrow 0$. Portanto $A \rightarrow 1 / \beta$ quando $\tau \rightarrow \infty$, fixando $B=0$. Além disso, fixando $A=0$, tem-se $B \rightarrow 1 / \beta$, quando $\tau \rightarrow \infty$. Deste modo, o círculo referido em (ii)(a) é o círculo de raio $1 / \beta$, centrado em $(0,0)$.

(b) Quando $\tau \rightarrow 0$, o vetor $\left[\cos \left(b_{1} \tau\right),-\operatorname{sen}\left(b_{1} \tau\right)\right] \rightarrow(1,0)$ ao longo do círculo unitário, no quarto quadrante. Deste modo, a condição de ortogonalidade $\left(1, b_{1}\right) \cdot\left[\cos \left(b_{1} \tau\right),-\operatorname{sen}\left(b_{1} \tau\right)\right]=0$, implica que $b_{1} \rightarrow \infty$ quando $\tau \rightarrow 0$. Veja a figura 3.1. Assim, dado um ponto qualquer $(A, B), \operatorname{com} A<1 / \beta, B>0$, existe $\tau$ suficientemente pequeno, de modo que $(A, B)$ está na região de estabilidade. Em virtude da simetria dessa região, segue-se (ii)(b). 
Na figura 3.4, esboçamos a região de estabilidade para alguns valores do retardamento.

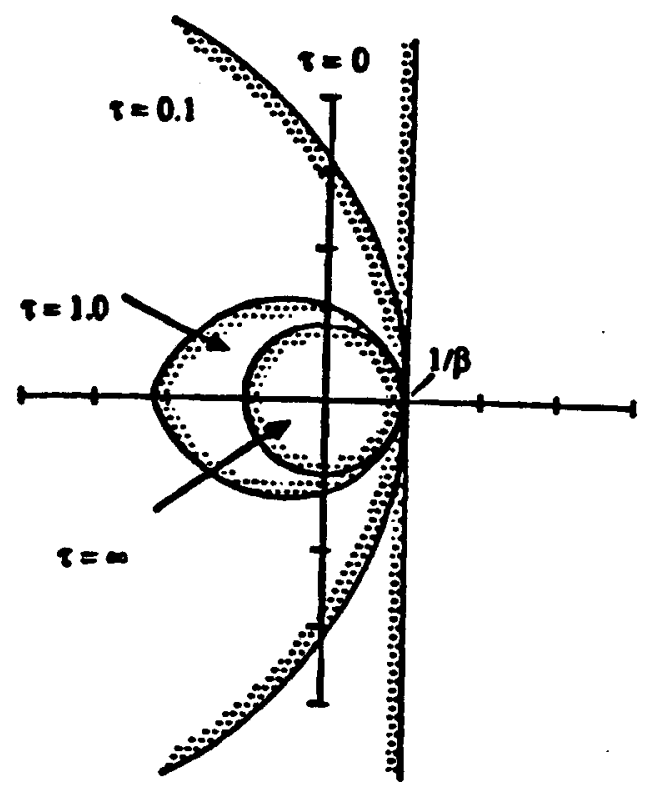

Figura 3.4: Região de estabilidade no $\lambda$-plano complexo

Lema 3.2 Quando a matriz de conexão $\left(J_{i j}\right)$, do sistema (2.4) é simétrica e $\tau$ é suficientemente pequeno, a origem será assintoticamente estável quando $\lambda_{\max }<$ $1 / \beta$ e $\lambda_{\min }>-(1 / \beta)(\pi / 2 \tau)$.

Prova: Os valores de $\alpha$ onde ocorrem as bifurcações do tipo "pitchfork" e de Hopf, são respectivamente, $\alpha=e^{\tau}$ e $\alpha=-b e^{\tau} / \operatorname{sen}(b \tau)$, onde $b \cot (b \tau)=-1$, $0<b<\pi / \tau$. Por outro lado, $\alpha=\beta \lambda e^{\tau}$. Assim, a origem será assintoticamente estável se $\lambda_{\max }<1 / \beta$ e $\lambda_{\min }>-(1 / \beta)(\pi / 2 \tau)$.

A figura 3.5 mostra a evolução da região de estabilidade da origem, $\operatorname{com} \tau$ fixo, para valores diferentes de $\beta$. Sendo $b_{1}$ e $b_{2}$ como na prova das afirmaçóes (3.1) e (3.2), portanto limitados, podemos observar que em vista de (3.7) e (3.10), que a região de estabilidade decresce na mesma ordem que $1 / \beta$, quando $\beta$ cresce. Cada construção também mostra esquematicamente uma distribuição de autovalores para dois tipos de redes simétricas: $\left|\lambda_{\max } / \lambda_{\min }\right|<1$ e $\left|\lambda_{\max } / \lambda_{\min }\right|>1$, onde $\lambda_{\max }$ e $\lambda_{\min }$ são respectivamente, o maior e o menor autovalor de conexão.

Para pequenos valores de $\beta$, todos os autovalores de conexão pertencem ao interior da região de estabilidade e a origem é o único ponto fixo, que é estável, 
como na figura 3.5 a e 3.5 b. À medida que $\beta$ cresce, o primeiro autovalor de conexão a deixar a região de estabilidade será:

(a) $\lambda_{\min }$, como na figura $3.5 \mathrm{c}$, dando origem a uma bifurcação de Hopf em $\lambda=-(1 / \beta) \sqrt{1+b_{2}^{2}}$, com o aparecimento de uma solução periódica estável.

(b) $\lambda_{\max }$, como na figura $3.5 \mathrm{~d}$, com dois pontos fixos aparecendo, um em cada lado da origem, bifurcação tipo "pitchfork".

0 valor de $\beta$ em que $\lambda_{\min }$ deixa a região de estabilidade na figura $3.5 \mathrm{c}$ é dado por:

$$
\beta=-\frac{\sqrt{1+b^{2}}}{\lambda_{\min }},
$$

onde $b=-\tan (b \tau), \pi / 2<b \tau<\pi$. No limite do menor retardamento, esse valor de $\beta$ é dado por:

$$
\beta \simeq-\frac{\pi}{2 \tau \lambda_{\min }}
$$

Decorre da estimativa do período formulada no Teorema da bifurcação de Hopf que o período de oscilação é aproximadamente $2 \pi / b \simeq 4 \tau$, para $\tau$ suficientemente pequeno.

Para uma distribuição de autovalores de conexão que satisfaz $\left|\lambda_{\max } / \lambda_{\min }\right|<1$, a primeira bifurcação a ocorrer, quando $\beta$ cresce, pode ser uma bifurcação do tipo "pitchfork", se $\lambda_{\max }$ deixa a região de estabilidade, ou uma bifurcação de Hopf, se $\lambda_{\min }$ deixa a região de estabilidade, dependendo do valor do retardamento, $\tau$. Para uma distribuição de autovalores que satisfaz $\left|\lambda_{\max } / \lambda_{\min }\right|>1, \lambda_{\max }$ sempre deixará a região de estabilidade antes que $\lambda_{\min }$, independentemente do valor do retardamento.

Um critério de estabilidade para redes simétricas, com ganho $\beta$ suficientemente pequeno, de modo que $\beta<1 / \lambda_{\max }$, baseado na análise da estabilidade linear, pode ser formulado exigindo-se que $\lambda_{\min }$, permaneça dentro da fronteira negativa da região de estabilidade da origem, ou seja,

$$
\lambda_{\min }>-(1 / \beta) \sqrt{1+b^{2}},
$$

onde $b=-\tan (b \tau), \pi / 2<b \tau<\pi$. Como esse valor é sempre maior que seu limite $\pi / 2 \tau$, o critério pode assim ser estabelecido:

$$
\tau<-\frac{\pi}{2 \beta \lambda_{\min }}
$$




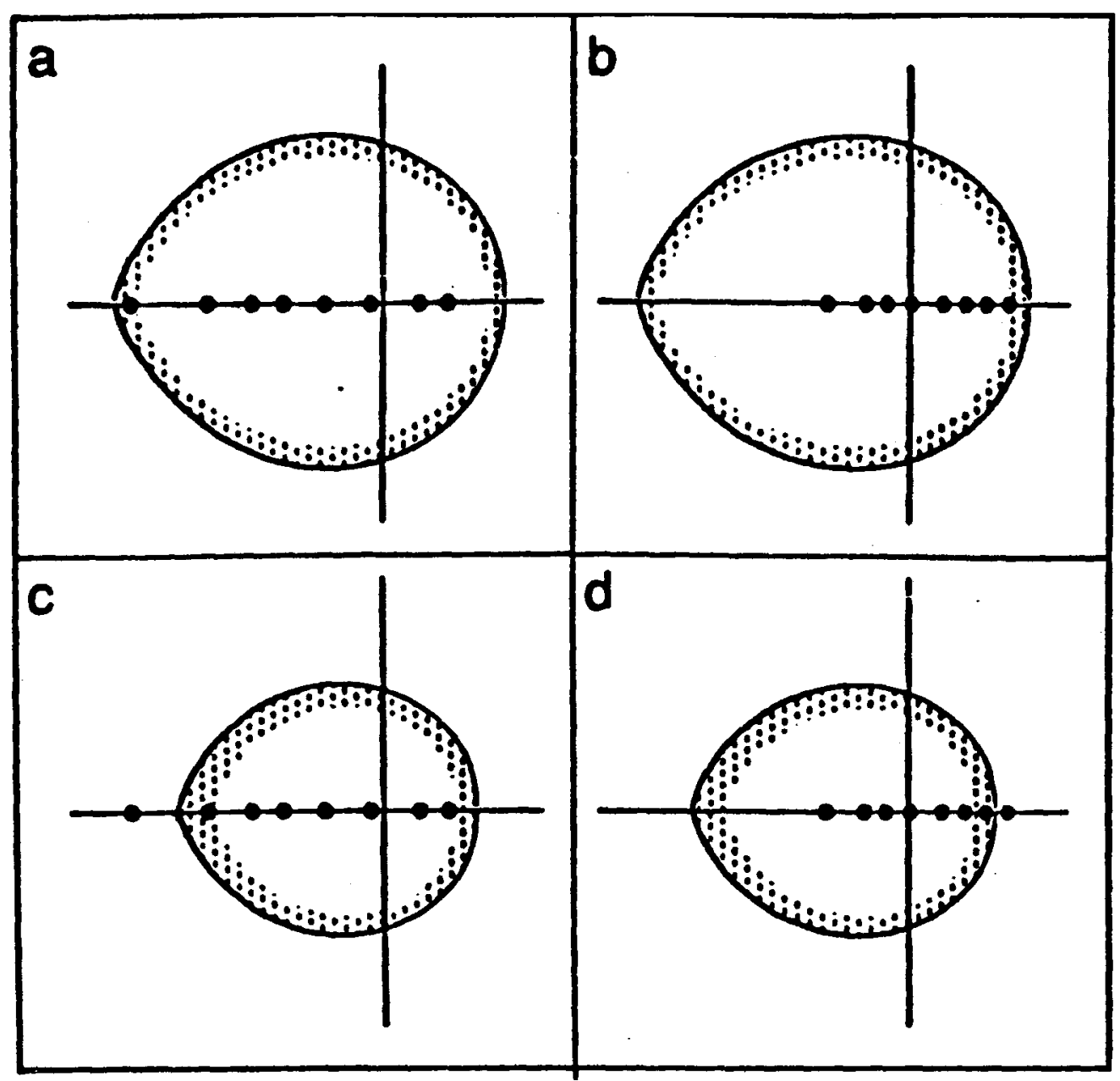

Figura 3.5: Variação da região de estabilidade com o ganho 


\section{Capítulo 4}

\section{Um caso de matriz de conexão não simétrica}

Neste capítulo trataremos de um caso não englobado pelo estudo de C.M. Marcus e R.M. Westervelt [7].

\subsection{Um critério de estabilidade em termos do ganho}

Como já observamos no capítulo 2, C.M. Marcus e R.M. Westervelt consideram, na verdade, um problema escalar, uma vez que supōem a matriz $\left(J_{i j}\right)$ diagonalizável.

Sem atingirmos a mais ampla generalidade, o que faremos a seguir é estender o nosso estudo para o sistema

$$
\dot{u}_{i}(t)=-u_{i}(t)+\sum_{j=1}^{n} J_{i j} f\left(u_{j}(t-\tau)\right),
$$

onde, $i=1, \ldots, n$, a matriz $J_{i j}$ é uma matriz diagonal de blocos da forma

$$
K_{l}=\left[\begin{array}{cc}
0 & \lambda_{l} \\
-\lambda_{l} & 0
\end{array}\right] \text { ou } K_{l}=\left[\begin{array}{cc}
0 & -\lambda_{l} \\
\lambda_{l} & 0
\end{array}\right],
$$

onde $\lambda_{l} \in \mathbf{R} \backslash\{0\}, l=1, \ldots, p$, ou ainda,

$$
J_{i j}=\operatorname{diag}\left(K_{1}, K_{2}, \ldots, K_{p}, \lambda_{p+1}, \ldots, \lambda_{n}\right) .
$$


Podemos olhar para o sistema em $p$ subespaços de dimensão 2 e $n-p$ subespaços de dimensão 1, estudando-os separadamente. 0 caso dos $n-p$ subespaços de dimensão 1, já foi tratado no capítulo 2 e o sistema para um subespaço de dimensão 2 torna-se:

$$
\left\{\begin{array}{l}
\dot{u}_{1}(t)=-u_{1}(t)+\lambda f\left(u_{2}(t-\tau)\right) \\
\dot{u}_{2}(t)=-u_{2}(t)-\lambda f\left(u_{1}(t-\tau)\right) .
\end{array}\right.
$$

Podemos descrever este sistema 2 por 2 pela equação

$$
\dot{x}(t)=-x(t)+\lambda F(x(t-\tau)),
$$

onde, $x=\operatorname{col}\left(x_{1}, x_{2}\right) \in \mathbf{R}^{2}, F: \mathbf{R}^{2} \rightarrow \mathbf{R}^{2}, F \in C^{3}, F(x)=\left(F_{1}(x), F_{2}(x)\right)=$ $\left(f\left(x_{2}\right),-f\left(x_{1}\right)\right)$, e $f$ é uma função "sigmoidal", com $f^{\prime}(0)=\beta>0$, como na equação (2.4), capítulo 2 .

Desta forma, a seguinte condição está satisfeita: $(\mathrm{H}) x_{2} F_{1}(x)>0, x_{2} \neq 0$ e $x_{1} F_{2}(x)<0, x_{1} \neq 0$.

Considerando $\beta$ como parâmetro, vamos mostrar que existe uma bifurcação de Hopf em um valor crítico $\beta_{0}$. Para fazer isso, vamos estudar os autovalores da equação linearizada de

$$
\dot{x}(t)=-x(t)+\lambda F(x(t-\tau)),
$$

ou seja,

$$
\left\{\begin{array}{l}
\dot{x}_{1}(t)=-x_{1}(t)+\lambda \beta x_{2}(t-\tau) \\
\dot{x}_{2}(t)=-x_{2}(t)-\lambda \beta x_{1}(t-\tau) .
\end{array}\right.
$$

$\mathrm{Se}$

$$
B(\beta)=\left[\begin{array}{cc}
0 & \beta \\
-\beta & 0
\end{array}\right]
$$

a linearização de (4.3) próxima à origem nos dá

$$
\dot{x}(t)=-x(t)+\lambda B(\beta) x(t-\tau) .
$$

Uma condição necessária e suficiente para a existência de uma solução não trivial de (4.4) da forma $x(t)=e^{s t} c, c \in \mathbf{R}^{2}$, é que $c$ seja uma solução não nula de

$$
s e^{s t} c=-e^{s t} c+\lambda B(\beta) e^{e t} e^{-s t} c,
$$

ou seja,

$$
\left[(s+1) I-\lambda B(\beta) e^{-s \tau}\right] c=0 .
$$


Isto quer dizer que

$$
\operatorname{det}\left[\begin{array}{cc}
s+1 & -\beta \lambda e^{-s \tau} \\
\beta \lambda e^{-s \tau} & s+1
\end{array}\right]=0
$$

implicando que,

$$
(s+1)^{2}+\lambda^{2} \beta^{2} e^{-2 s \tau}=0,
$$

ou ainda,

$$
(s+1)^{2} e^{2 s \tau}=-\lambda^{2} \beta^{2} .
$$

Esta equação em $s$ é a chamada equação característica de (4.4). Fazendo uma mudança do parâmetro $\beta, \alpha=\beta \lambda e^{\tau}$ e uma translação de $s, \mu=s+1$, temos

$$
\mu^{2} e^{2 \mu \tau}=-\alpha^{2} \text {. }
$$

Observação $4.1 \mu=0$ é a única raiz real de (4.6) e ocorre quando $\alpha=0$. Mais ainda, $\mu$ é raiz de (4.6) se, e só se, seu complexo conjugado $\bar{\mu}$ também o é. Portanto, restringimos nosso estudo ao semiplano superior $\Im(\mu)>0$.

Lema 4.1 As raizes imaginárias puras de (4.6) são $\mu=(k \pi / \tau) i$, e somente ocorrem para $\alpha= \pm k \pi / \tau, k=0,1, \ldots$

Prova: Substituindo $\mu=a+b i$, na equação (4.6) obtemos,

$$
(a+b i)^{2} e^{2(a+b i) r}=-\alpha^{2},
$$

ou seja,

$$
(a+b i)^{2} e^{2 a \tau}[\cos (2 b \tau)+i \operatorname{sen}(2 b \tau)]=-\alpha^{2},
$$

ou ainda,

$$
\left(a^{2}+2 a b i-b^{2}\right) e^{2 a \tau}[\cos (2 b \tau)+i \operatorname{sen}(2 b \tau)]=-\alpha^{2} .
$$

Igualando as partes reais e imaginárias obtemos o seguinte sistema

$$
\left\{\begin{array}{l}
e^{2 a \tau}\left[\left(a^{2}-b^{2}\right) \cos (2 b \tau)-2 a b \operatorname{sen}(2 b \tau)\right]=-\alpha^{2} \\
\left(a^{2}-b^{2}\right) \operatorname{sen}(2 b \tau)+2 a b \cos (2 b \tau)=0 .
\end{array}\right.
$$

Assumindo $\mu=b i, b>0$, o sistema (4.7) torna-se:

$$
\left\{\begin{array}{l}
-b^{2} \cos (2 b \tau)=-\alpha^{2} \\
-b^{2} \operatorname{sen}(2 b \tau)=0
\end{array}\right.
$$

ou ainda,

$$
\left\{\begin{array}{l}
\cos (2 b \tau)=\alpha^{2} / b^{2} \\
\operatorname{sen}(2 b \tau)=0 .
\end{array}\right.
$$

Como $b>0$ e $\cos (2 b \tau)>0$, concluímos que $b=k \pi / \tau$ e $\alpha= \pm k \pi / \tau, k=0,1, \ldots \square$ 
Lema 4.2 Não existe raiz de (4.6) nas retas $a+b i$, onde $b=\left(k+\frac{1}{2}\right) \frac{\pi}{7}$, $k=0,1, \ldots$

Prova: Se $\mu=a+\left(\frac{k}{\tau}+\frac{1}{2 \tau}\right) \pi i$ o sistema (4.7) torna-se:

$$
\left\{\begin{array}{l}
e^{2 a \tau}\left[a^{2}-\left(\frac{k}{\tau}+\frac{1}{2 \tau}\right)^{2} \pi^{2}\right] \cos \left[2\left(k+\frac{1}{2}\right) \pi\right]=-\alpha^{2} \\
2 a\left(\frac{k}{\tau}+\frac{1}{2 \tau}\right) \pi \cos [(2 k+1) \pi]=0,
\end{array}\right.
$$

ou seja,

$$
\left\{\begin{array}{l}
e^{2 a r}\left[a^{2}-\left(\frac{k}{\tau}+\frac{1}{2 \tau}\right)^{2} \pi^{2}\right]=\alpha^{2} \\
2 a\left(\frac{k}{\tau}+\frac{1}{2 \tau}\right) \pi=0 .
\end{array}\right.
$$

Da segunda equação obtemos $a=0$. Substituindo na primeira equação obtemos

$$
-\left(\frac{k}{\tau}+\frac{1}{2 \tau}\right)^{2} \pi^{2}=\alpha^{2}
$$

Logo essas equações são incompatíveis.

Este lema afirma que as raízes $\mu$ da equação (4.6) estão distribuidas em algumas das faixas:

$$
\begin{gathered}
S_{0}=\left\{\mu=a+b i: 0<b<\frac{\pi}{2 \tau}\right\}, \\
S_{k}=\left\{\mu=a+b i:\left(k-\frac{1}{2}\right) \frac{\pi}{\tau}<b<\left(k+\frac{1}{2}\right) \frac{\pi}{\tau}\right\}, k=1,2, \ldots
\end{gathered}
$$

Lema 4.3 Se $\mu=a+b i \in S_{0}, \mu \neq 0$, é uma raiz de (4.6), então $a>0$.

Prova: Segue da equação (4.6) que $\mu e^{\mu \tau}$ é um número imaginário puro, ou seja,

$$
\mu e^{\mu \tau}= \pm \alpha i \text {. }
$$

Sendo que

$$
\Re\left(\mu e^{\mu \tau}\right)=e^{a \tau}[a \cos (b \tau)-b \operatorname{sen}(b \tau)]=0,
$$

temos

$$
a=\frac{b \operatorname{sen}(b \tau)}{\cos (b \tau)} \text {. }
$$

Como $\cos (b \tau)>0$ e $b \operatorname{sen}(b \tau)>0$, concluímos que $a$ deve ser positivo. 
Lema 4.4 Cada faixa $S_{k}, k=0,1,2, \ldots$, contém precisamente uma raiz de (4.6).

Prova: Daremos a prova para a faixa $S_{1}$. Seja $\mu=a+b i \in S_{1}$ e $\alpha>0$. A equação (4.6) implica que

$$
\Re\left(\mu e^{\mu \tau}\right)=e^{a \tau}[a \cos (b \tau)-b \operatorname{sen}(b \tau)]=0,
$$

e sendo que $\cos (b r)<0$, temos

$$
a \operatorname{sen}(b \tau)=\frac{b \operatorname{sen}^{2}(b \tau)}{\cos (b \tau)}<0
$$

Logo

$$
\Im\left(\mu e^{\mu \tau}\right)=e^{a \tau}[b \cos (b \tau)+a \operatorname{sen}(b \tau)]<0
$$

$e$, portanto, na faixa em questão (4.6) é equivalente à

$$
\mu e^{\mu r}=-i \alpha
$$

e (4.8) pode ser reescrita como

$$
\left\{\begin{array}{l}
e^{a \tau}[a \cos (b \tau)-b \operatorname{sen}(b \tau)]=0 \\
e^{a \tau}[a \operatorname{sen}(b \tau)+b \cos (b \tau)]=-\alpha
\end{array}\right.
$$

o que implica

$$
\left\{\begin{array}{l}
a=b \tan (b \tau) \\
e^{a \tau}[a \operatorname{sen}(b \tau)+b \cos (b \tau)]=-\alpha .
\end{array}\right.
$$

Substituindo a primeira equação de (4.9) na segunda obtemos:

$$
e^{b \tau \tan (b \tau)}[b \tan (b \tau) \operatorname{sen}(b \tau)+b \cos (b \tau)]=-\alpha,
$$

ou seja,

$$
\alpha=\frac{-b e^{b \tau \tan (b \tau)}}{\cos (b \tau)} \stackrel{\text { def }}{=} \Lambda(b) .
$$

Com algum trabalho de rotina pode-se preencher os detalhes dos seguintes cálculos.

$$
\begin{aligned}
\frac{d \Lambda}{d b} & =\frac{\left\{-e^{b \tau \tan (b \tau)}-b e^{b \tau \tan (b \tau)}\left[\tau \tan (b \tau)+b \tau^{2} \sec ^{2}(b \tau)\right]\right\} \cos (b \tau)}{\cos ^{2}(b \tau)}- \\
& =-e^{b \tau \tan (b \tau)}\left[(1+b \tau \tan (b \tau))^{2}+b^{2} \tau^{2}\right] \sec (b \tau)>0,
\end{aligned}
$$


e ainda,

$$
\Lambda\left(\frac{\pi}{2 \tau}+\right)=0, \Lambda\left(\frac{3 \pi}{2 \tau}-\right)=+\infty
$$

Seja agora $\mu=a+b i \in S_{1}$ e $\alpha<0$. A equação (4.6) implica que

$$
\Re\left(\mu e^{\mu \tau}\right)=e^{a \tau}[a \cos (b \tau)-b \operatorname{sen}(b \tau)]=0,
$$

e sendo que $\cos (b \tau)<0$, temos

$$
a \operatorname{sen}(b r)=\frac{b \operatorname{sen}^{2}(b \tau)}{\cos (b \tau)}<0
$$

Logo

$$
\Im\left(\mu e^{\mu \tau}\right)=e^{a \tau}[b \cos (b \tau)+a \operatorname{sen}(b \tau)]<0
$$

e, portanto, na faixa em questão, (4.6) é equivalente à

$$
\mu e^{\mu \tau}=i \alpha,
$$

e (4.12) pode ser reescrita como

$$
\left\{\begin{array}{l}
a=b \tan (b \tau) \\
e^{a \tau}[a \operatorname{sen}(b \tau)+b \cos (b \tau)]=\alpha .
\end{array}\right.
$$

Substituindo a primeira equação de (4.13) na segunda obtemos:

$$
e^{b \tau \tan (b \tau)}[b \tan (b \tau) \operatorname{sen}(b \tau)+b \cos (b \tau)]=\alpha,
$$

ou seja,

$$
\alpha=\frac{b e^{b \tau \tan (b \tau)}}{\cos (b \tau)} \stackrel{\text { def }}{=} \Lambda(b) .
$$

Assim

$$
\frac{d \Lambda}{d b}=e^{b \tau \tan (b \tau)}\left[(1+b \tau \tan (b \tau))^{2}+b^{2} \tau^{2}\right] \sec (b \tau)<0
$$

e

$$
\Lambda\left(\frac{\pi}{2 \tau}+\right)=0, \Lambda\left(\frac{3 \pi}{2 \tau}-\right)=-\infty
$$

As propriedades de $\Lambda$ implicam que (4.10) define $b$ univocamente como função de $\alpha, 0<\alpha<+\infty$. Conforme a equação (4.14), $b$ também fica univocamente definido como função de $\alpha,-\infty<\alpha<0$. Através da primeira equação de (4.9) [ou(4.13)], a também fica definido como função de $\alpha, 0<\alpha<+\infty$ [ou $-\infty<\alpha<0$ ], e portanto $\mu \in S_{1}$ é uma função de $\alpha$. Mais ainda, $\mu$ é diferenciável em relação à $\alpha$.

Através de raciocínio completamente análogo, chega-se à mesma conclusão para cada uma das faixas $S_{k}, k=0,1,2, \ldots$ 
Lema 4.5 Toda raiz de (4.6) é simples.

Prova: Seja $\mu_{0}$ raiz de (4.6). Desde que $\mu_{0} \neq-1 / \tau$, obtemos

$$
\frac{d}{d \mu}\left(\mu^{2} e^{2 \mu \tau}+\alpha^{2}\right)_{\mu=\mu_{0}}=2 \mu_{0} e^{2 \mu_{0} \tau}\left(1+\mu_{0} \tau\right) \neq 0
$$

e portanto $\mu_{0}$ é simples.

Observação 4.2 A prova do Lema 4.4 contém alguns fatos importantes que serão destacados a seguir.

1. Se $\mu$ permanece no semiplano superior, $\Im(\mu)>0$, em cada faixa $S_{k}$, a raiz $\mu$ é uma função diferenciável de $\alpha$.

2. Como $\frac{d b}{d \alpha}$ tem o mesmo sinal de $\alpha$, em virtude de (1.11) e (4.15) e, além disso, como

$$
|\tan (b \tau)|<b \tau \sec ^{2}(b \tau),
$$

segue que

$$
\frac{d a}{d \alpha}=\left(\tan (b \tau)+b \tau \sec ^{2}(b \tau)\right) \frac{d b}{d \alpha}
$$

também tem o sinal de $\alpha$.

Lema 4.6 Se $\mu_{k}=a_{k}+b_{k} i \in S_{k}, k=0,1, \ldots$, são raizes de (4.6), para algum $\alpha$, então suas partes reais satisfazem $a_{0}>a_{1}>\ldots \rightarrow-\infty$.

Prova: Se $\mu=a+b i$, a equação (4.6) implica que as raízes $\mu_{k}, k=0,1, \ldots$ devem satisfazer a condição

$$
\left(a^{2}+b^{2}\right) e^{2 a \tau}=\alpha^{2}
$$

e essa, para $\alpha=\pi / \tau$, define $b>0$ como função de $a$ no intervalo $-\infty<a \leq 0$. De fato,

$$
a^{2}+b^{2}=\frac{\pi^{2}}{\tau^{2}} e^{-2 a \tau},
$$

o que implica

$$
b=\left[\left(\frac{\pi}{\tau} e^{-a \tau}-a\right)\left(\frac{\pi}{\tau} e^{-a \tau}+a\right)\right]^{1 / 2} .
$$

Temos que verificar que

$$
G(a) \stackrel{\operatorname{def}}{=} \frac{\pi}{\tau} e^{-a \tau}+a
$$


é positiva para $a \leq 0$. Mas $G(0)=\pi / \tau, G^{\prime}(a)=-\pi e^{-a \tau}+1<0$ e portanto $G(a)>0$ para $a \leq 0$. Então, de acordo com os lemas 4.1 e 4.3, $a_{0}>a_{1}=0>a_{k}$, $k=2,3, \ldots$ Como

$$
\frac{d b}{d a}=-\frac{1}{b}\left(\frac{\pi^{2}}{\tau} e^{-2 a \tau}+a\right)<0
$$

para $a \leq 0$, segue que os $a_{k}^{\prime} s$ são estritamente decrescentes em relação aos $b_{k}^{\prime} s$. Assim, a ordem especificada das partes reais das raizes ocorre pelo menos no caso em que $\alpha=\pi / \tau$.

Suponhamos que o lema seja falso para algum valor do parâmetro $\alpha$. Então recordando que cada $a_{k}$ é uma função contínua de $\alpha$, existe algum $\alpha=\hat{\alpha}$, para o qual a equação (4.6) tem raízes $\mu_{k}$ e $\mu_{l}$ tais que $a_{k}=a_{l}=a, k>l$. Mas, como $b_{k}>b_{l}$, a equação (4.16) leva-nos à contradição

$$
\left(a^{2}+b_{k}^{2}\right) e^{2 a \tau}=\left(a^{2}+b_{l}^{2}\right) e^{2 a \tau} .
$$

Assim $a_{0}>a_{1}>\ldots$

Fazendo $a=a_{k}, b=b_{k}$ em (2.9), vemos que $a_{k} \rightarrow-\infty$ quando $k \rightarrow \infty$.

Recordando que o lugar das raízes $\mu$ da equação (4.6) é simétrico em relação ao eixo real, os lemas mostram uma descrição completa deste. Quando $\alpha$ varia de 0 até $+\infty$ ou 0 até $-\infty$, as raízes de (4.6) descrevem as curvas

$$
a=b \tan (b \tau)
$$

em $S_{k}, k=0,1, \ldots$ com $a$ crescente. A figura 4.1 mostra uma situação particular para um valor de $\alpha$ fixado.

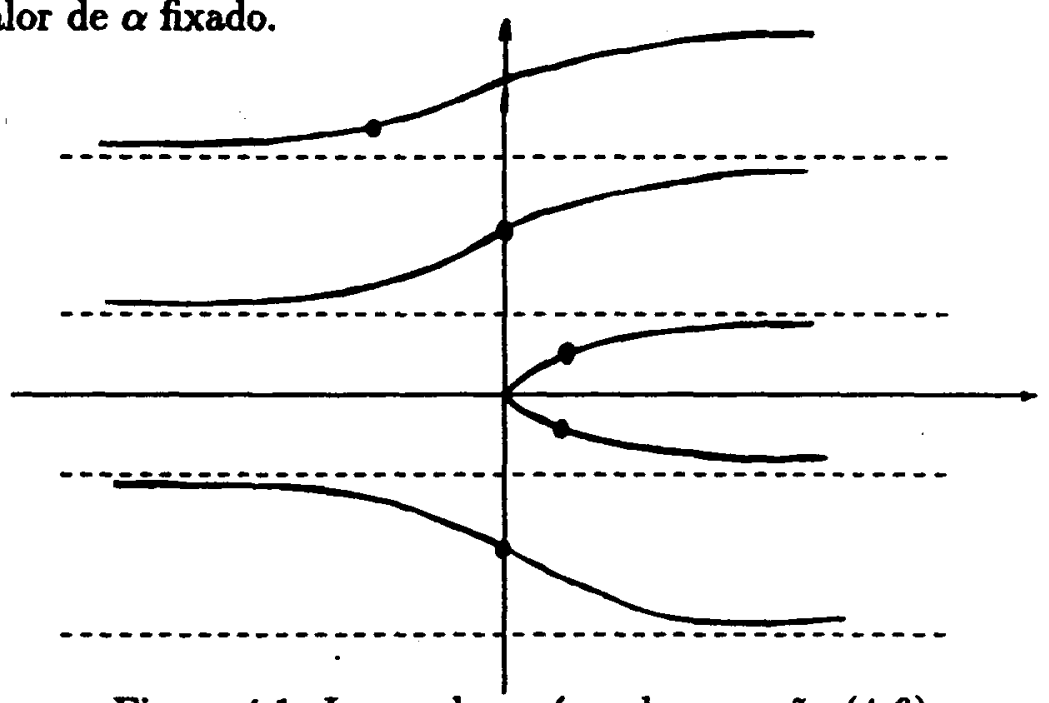

Figura 4.1: Lugar das raízes da equação (4.6) 
A maneira que as raízes $\mu$ estão distribuídas nas curvas $a=b \tan (b \tau)$ é coerente com a ordem das partes reais estabelecidas no lema 4.6. Quando $\beta$ cruza um certo valor $\beta_{0}$ da esquerda para a direita, um par de raízes características conjugadas $s_{0}=s\left(\beta_{0}\right), \bar{s}_{0}$, cruza o eixo imaginário pela primeira vez, da esquerda para a direita.

Como o autovalor de (4.5) é a translação $s=\mu-1$ das raízes $\mu$ da equação (4.6), $s_{0}$ corresponde ao valor $\beta_{0}$ de $\beta$, associado a $\mu=\mu_{0} \in S_{0}$ da forma $\mu_{0}=$ $1+b i$, ou seja,

$$
\Re\left(\mu e^{\mu \tau}\right)=e^{\tau}[\cos (b \tau)-b \operatorname{sen}(b \tau)]=0 .
$$

Como $\cos (b r)>0$ na faixa $S_{0}$ e $a=b \tan (b r)$, vem

$$
a \operatorname{sen}(b \tau)=\frac{b \operatorname{sen}^{2}(b \tau)}{\cos (b \tau)}>0 .
$$

Logo,

$$
\Im\left(\mu e^{\mu \tau}\right)=e^{a \tau}[b \cos (b \tau)+a \operatorname{sen}(b \tau)]>0
$$

e, portanto, fixando-nos no caso $\alpha>0$, podemos escrever na faixa $S_{0}$ :

$$
\mu e^{\mu \tau}=i \alpha \text {. }
$$

Assim, fazendo $\mu=\mu_{0}$,

$$
\left\{\begin{array}{l}
\Re\left(\mu e^{\mu \tau}\right)=e^{\tau}[\cos (b \tau)-b \operatorname{sen}(b \tau)]=0 \\
\Im\left(\mu e^{\mu \tau}\right)=e^{\tau}[\operatorname{sen}(b \tau)+b \cos (b \tau)]=\alpha_{0} .
\end{array}\right.
$$

Da primeira equação de (4.18) temos que

$$
b \operatorname{sen}(b \tau)=\cos (b \tau) .
$$

Substituindo na segunda equação temos:

$$
e^{\tau}\left[\operatorname{sen}(b \tau)+b^{2} \operatorname{sen}(b \tau)\right]=\alpha_{0},
$$

o que implica que,

$$
e^{\tau}\left[\left(1+b^{2}\right) \operatorname{sen}(b \tau)\right]=\alpha_{0} .
$$

Como $\alpha=\beta \lambda e^{\top}$ temos

$$
e^{\tau}\left(1+b^{2}\right) \operatorname{sen}(b \tau)=e^{\tau} \lambda \beta_{0},
$$

ou seja,

$$
\beta_{0}=\frac{\left(1+b^{2}\right) \operatorname{sen}(b \tau)}{\lambda},
$$

onde $b$ é dado por $b \tan (b \tau)=1,0<b<\pi / 2 \tau$.

Observar que o caso $\alpha<0$ é análogo, isto é, obtemos o mesmo $\beta_{0}$. 
Observação 4.3 Como $\alpha=\beta \lambda e^{\tau}$, segue que $\frac{d a}{d \beta}, \frac{d b}{d \beta}>0$ e, portanto, ao variar $\beta$, sempre que s cruza o eixo imaginário, o faz transversalmente.

Estamos agora em posição de estabelecer um interessante teorema de bifurcação de soluções periódicas para a equação (4.3). Trata-se de uma consequência dos lemas anteriores combinados com o Teorema 1.2 do capítulo 1 , estabelecendo uma sequência infinita de valores do ganho, $\beta_{0}<\beta_{1}<\ldots \rightarrow \infty$, onde ocorrem bifurcações de Hopf.

Teorema 4.1 Se (H) está satisfeita, então existe uma sequência infinita de valores do ganho, $\beta_{0}<\beta_{1}<\ldots \rightarrow \infty$, tal que a equação (4.3) tem uma bifurcação de Hopf em $\beta=\beta_{k}, k=0,1, \ldots$

Prova: Para provar este teorema, vamos verificar as hipóteses do Teorema da bifurcação de Hopf 1.2.

A sequência $\beta_{0}<\beta_{1}<\ldots$ corresponde aos valores do ganho, para os quais um par de raizes características conjugadas cruza o eixo imaginário. Sua monotonicidade segue da ordem das partes reais dos $s_{k}^{\prime} s$, dada no Lema 4.6 e da monotonicidade das partes reais de $s$ em relação à $\beta$, que segue da observação.

Como o espectro de uma equação diferencial linear com retardamento tem infinitas raízes características do lado esquerdo do eixo imaginário, temos que $\beta_{k} \rightarrow \infty$ quando $k \rightarrow \infty$.

O teorema é uma consequência imediata das seguintes afirmações, as quais saem diretamente dos lemas e observações anteriores.

(i) As raizes caracteristicas imaginárias puras $s_{k}=s_{k}\left(\beta_{k}\right) \neq 0, k=0,1, \ldots$ são simples, e para qualquer $k$ fixado, os autovalores $s_{j} \neq s_{k}, \bar{s}_{k}$ satisfazem $s_{j} \neq m s_{k}$, para qualquer inteiro $m$.

(ii) $\frac{d}{d \beta}\left[\Re\left(s_{k}(\beta)\right)\right]_{\beta=\beta_{k}} \neq 0$.

Um critério de estabilidade em relação ao ganho $\beta$, baseado na análise da estabilidade linear, pode ser formulado exigindo-se que $\beta<\beta_{0}$, ou seja,

$$
\beta<\frac{\left(1+b^{2}\right) \operatorname{sen}(b \tau)}{\lambda},
$$

onde $b \tan (b \tau)=1,0<b<\pi / 2 \tau$. 


\subsection{Um critério de estabilidade em termos do retardamento}

Pela mudança da escala do tempo $t=\tau t^{\prime}$, a equação (4.2) se transforma em

$$
v^{\prime}\left(t^{\prime}\right)=-\tau v\left(t^{\prime}\right)+\tau \lambda F\left(v\left(t^{\prime}-1\right)\right),
$$

onde $v=\operatorname{col}\left(v_{1}, v_{2}\right) \in \mathbf{R}^{2}, F \in C^{3}, F(v)=\left(f\left(v_{2}\right),-f\left(v_{1}\right)\right)$, e $f$ satisfaz as condições assumidas em (4.2).

A equação linearizada correspondente fica

$$
v^{\prime}\left(t^{\prime}\right)=-\tau v\left(t^{\prime}\right)+\tau \lambda B v\left(t^{\prime}-1\right)
$$

onde

$$
B=\left[\begin{array}{cc}
0 & \beta \\
-\beta & 0
\end{array}\right]
$$

e a equação característica é

$$
(s+\tau)^{2} e^{2 s}=-\tau^{2} \delta^{2}
$$

$\operatorname{com} \delta=\lambda \beta$.

Considerando $\tau$ como parâmetro, existe uma bifurcação de Hopf em um valor crítico $\tau_{0}$.

De fato, para a equação (4.21) vale o seguinte teorema [1, Teorema 1.1, página 11].

Teorema 4.2 Suponhamos $\delta>1$. Então existe uma sequência $0<\tau_{0}<\tau_{1}<$ $\ldots \rightarrow+\infty$ de valores do parâmetro $\tau$ para os quais a equação (4.19) tem uma bifurcação de Hopf local em $\tau=\tau_{k}, \tau_{k}=b_{k} / \sqrt{\delta^{2}-1}$, onde $b_{k}=\operatorname{arcsen}(-1)^{k} / \delta \in$ $\left(\left(k-\frac{1}{2}\right) \pi,\left(k+\frac{1}{2}\right) \pi\right) \cap(0, \infty), k=0,1, \ldots$ O ramo que emana de $\tau_{k}$ representa soluçôes $\omega$-periódicas com $\omega$ próximo de $2 \pi / b_{k}$.

Assim um critério de estabilidade para $\beta>1 / \lambda$ em relação ao retardamento $\tau$, baseado na análise da estabilidade linear, pode ser formulado exigindo-se que

$$
\tau<\tau_{0}
$$

onde $\tau_{0}$ é o primeiro termo da sequência dada no Teorema (4.2). 


\section{Bibliografia}

[1] Baptistini, M. T.Z. Soluções periódicas de uma equação diferencial no plano com retardamento global. Tese de Doutorado apresentada ao ICMSC- USP, 1990.

[2] Bellman, R.; Cooke, K. Differential difference equations. Academic Press, 1963.

[3] Cohen M. A.; Grossberg S. IEEE Trans, v. SMC-13, n. 815, 1983.

[4] Hale, J. K. Theory of functional differential equations. New York, Springer, 1977.

[5] Hayes, N. D. Roots of the transcendental equation associated with a certain differential difference equation. J. London Math. Soc., v. 25, p. 226-232, 1950.

[6] Hopfield, J. J. Proc. National Academy of Sciences USA, v. 81, p. 3008, 1984.

[7] Marcus, C. M.; Westervelt, R. M. Stability of analog neural networks with delay. (pré print a ser publicado no Physical Review A).

[8] Onuchic, N. Equações Diferenciais com Retardamento. In: Colóquio Brasileiro de Matemática 8, Poços de Caldas, 1971. Rio de Janeiro, IMPA, 1971. p. 1-36.

[9] Táboas, P. Z. Periodic solutions of a planar delay equation. Proc. Royal Society Edinburgh, v. 116 A, p. 85-101, 1990. 